\title{
Mechanisms of calcium transport across the placenta: Review
}

\author{
Catarina Tivane $^{1}$, Marcio Nogueira Rodrigues ${ }^{1}$, Phelipe Oliveira Favaron ${ }^{1}$, \\ Antonio Chaves de Assis Neto ${ }^{1}$, Eduardo Harry Birgel Júnior ${ }^{2}$, Maria Angelica Miglino ${ }^{1^{*}}$ \\ ${ }^{1}$ Faculty of Veterinary Medicine and Animal Science, University of Sao Paulo, Sao Paulo, Brazil; \\ *Corresponding Author: miglino@usp.br \\ ${ }^{2}$ Faculty of Food Engineering and Animal Science, University of Sao Paulo, Sao Paulo, Brazil
}

Received 8 August 2012; revised 10 September 2012; accepted 22 September 2012

\begin{abstract}
Studies of calcium transfer across the placenta have been reviewed because of the physiological and nutritional importance of this mineral during pregnancy, especially in order to better understand its contribution to development of the fetal skeleton. The placental transfer of maternal calcium to the fetus represents a vital mechanism for fetal development and breastmilk production, yet little meaningful information is currently available regarding the biochemical mechanisms involved in this process. Once again, the use of different animal models as rodents, rabbit, sheep and bovine have demonstrate different mechanisms of calcium transport across the placenta and contribute to better understand its effects in both fetus and mother during the gestation. In relation to the transfer of calcium from the mother to fetus data suggest it occur via an active mechanism; thus calcium concentration is higher in fetus than in maternal blood. Despite conflicting reports, several investigators agreed that calcium concentration in the fetal blood is mainly regulated by fetal parathyroid hormone and plasma concentration of vitamin D3, a metabolite that plays a key role in calcium transport through the syncytial cells.
\end{abstract}

Keywords: Calcium; Transplacental; Homeostasis; Transport; Placenta

\section{INTRODUCTION}

A consider number of reports have demonstrated the importance of calcium for the embryo development and their physiological functions, including cellular division and differentiation for different cell types [1,2]. Among other micronutrients, calcium is an essential mineral cri- tical for normal health and development specially to maintain the fetus in a hypercalcaemic state relative to the mother. This condition has been thought to facilitate normal development of the fetal skeleton.

The mechanisms of calcium transport across the placenta have been studied in a number of domestic and wild mammals as well as in the human. The earlier descriptions by [3-7] represented some of the most comprehensive reports, which provided basis for later researches. Recently, the calcium channels, transporters and exchangers in the human placenta were reviewed by [1].

The plasmatic calcium can be found in three different forms: 1) bound to proteins, mainly albumin $(40 \%), 2)$ complexed with ions like bicarbonate (10\%) and 3$)$ the one which is "free" or ionized (50\%) [8].

Transplacental movement of calcium increases drastically during the last trimester of gestation in all studied species [4]. According to [7] the cellular mechanisms that mediate mineral transport in the enterocyte and in the trophoblast share features in common, although their humoral regulations are quite distinct. The author suggested three steps of mineral transport in both cell types: 1) uptake at the enterocyte/trophoblast microvillous membrane; 2) transcellular transport; and 3) extrusion at the basolateral membrane (BLM) into the fetal circulation.

The transfer of calcium from the mother to fetus occurs via an active mechanism [5] based on two observations: first, the calcium concentration is higher in the fetus than in maternal blood and, second, the perfusion in situ of the umbilical artery resulted in a net increase of the calcium concentration of the perfusate in the fetus. According to [7,9] the placental calcium transport appears to be regulated by parathyroid hormone related protein $(\mathrm{PTHr})$ rather than $1.25(\mathrm{OH}) 2 \mathrm{D}$ once that using experimental animals the placental calcium gradient was lost by removal of PTHrP by thyroparathyroidectomy. In this way, the calcium concentration was regulated by fetal 
parathyroid hormone and the plasma concentration of $1,25-(\mathrm{OH}) 2$ vitamin $\mathrm{D} 3$, which plays a key role in calcium transport through the syncytial cells [6].

Transcellullar calcium transport in renal distal tubules and in syncytiotrophoblasts of the placenta has been reported as being by an active mechanism [6]. According to [2] the syncytiotrophoblasts actively transport about $80 \%$ of calcium from maternal to fetal circulation. Although there is substantial information on various aspects of calcium transfer in a variety of species including humans, much of it remains separated by species. Therefore, we believed that a broader synthesis of this information across species would be useful, always taking account of diversity in placental morphology and function between species.

A full understanding of the mechanisms of calcium transport across the placenta requires answers to several questions including the following:

1) What are the magnitudes of unidirectional fluxes across the placenta, both maternofetal and fetomaternal?

2) What are the magnitudes and profiles of electrochemical gradients across the placenta?

3) What proportions of the fluxes are extracellular and what proportions transcellular, using routes transport proteins (carriers and channels) in the trophoblast plasma membrane?

4) What is the most common route of calcium transport in the different species?

5) Is there any reported mechanism of ionized $\mathrm{Ca}^{2+}$ transfer across the placenta?

6) Is there any control of placental ionized $\mathrm{Ca}^{2+}$ transfer and if so, how it is brought about?

\section{WHAT IS THE MAGNITUDE OF UNIDIRECTIONAL FLUXES ACROSS THE PLACENTA, BOTH MATERNOFETAL AND FETOMATERNAL?}

In the rat, mouse and hamster the chorioallantoic placenta is of the haemotrichorial type [10]. Like other haemochorial placentae, the rat placenta is much more permeable than the epitheliochorial type (Figure 1), i.e. in sheep placenta; it can be assumed that the transcellular transport of calcium is paralleled by a bidirectional diffusion movement through paracellular routes. The placental transport of calcium in the rat and mouse is highly asymmetrical, maternofetal prevailing [11,12], with only a small bidirectional diffusional component. Most of the maternofetal transport is transcellular and seems to be effected by primary active transport. In the rat, the unidirectional maternofetal flux of calcium is $100+/-7$ $\mathrm{nmol} \cdot \mathrm{min}^{-1}$. Therefore, the fetomaternal transfer of calcium is estimated as only $20 \%$ of the maternal-fetal transfer [11].

Previous experiments in order to understand the calcium transfer using the guinea-pig as a model showed that the calcium influx was always significantly lower on the maternal side than on the fetal side. The concentration on fetal side was $1.8+/-0.7 \mathrm{mM}$ and Vmax was $1.66+/-0.28 \mathrm{umol} / \mathrm{min} \cdot \mathrm{g}$ compared with 0.18 to 1.15 $\mathrm{mM}$ and $\mathrm{Vmax}$ from 0.12 to $0.50 \mathrm{umol} / \mathrm{min} \cdot \mathrm{g}$ on the maternal side [13]. Although Ca-ATPase activity has been demonstrated in placental membrane vesicles [13] it is not clear whether this enzyme is associated with placental calcium transport.

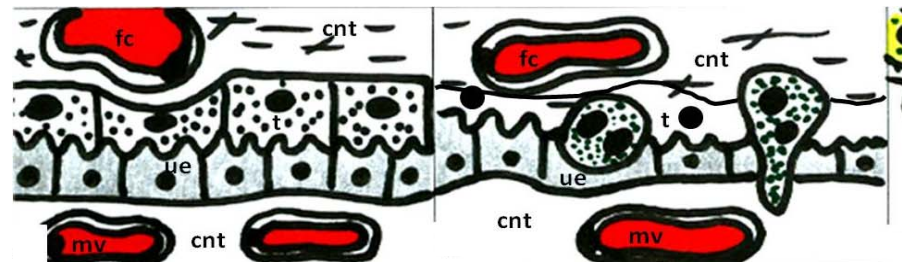

(a)

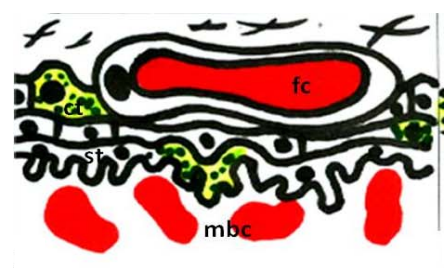

(d) (b)

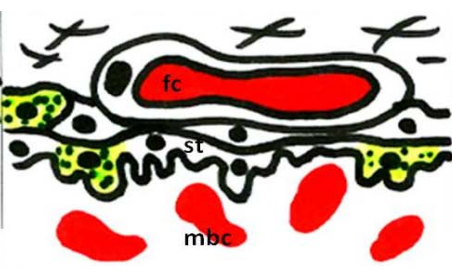

(e)

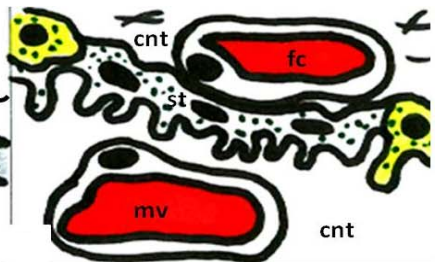

(c)

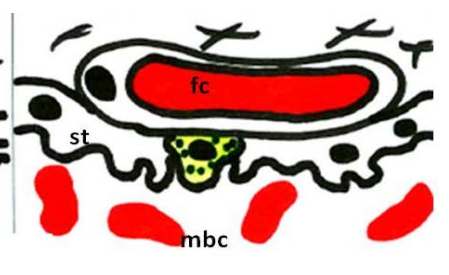

(f)

Figure 1. Schematic view of the different types of placental barrier in the domestic animals. (a) Epitheliochorial placenta (horse and pig); (b) Synepitheliochorial placenta (ruminants: sheep, cow and goat); (c) Endotheliochorial placenta (Carnivora); (d)-(f) Hemochorial placentae (rodents): (d) Haemotrichorial type (rats, mouse and mice), (e) Haemodichorial (rabbits) and (f) Haemomonochorial (Hystricomorph rodents and human). Legends: $\mathrm{fc}=$ fetal capillary, $\mathrm{cnt}=$ connective tissue, $\mathrm{t}=$ trophoblast, $\mathrm{ue}=\mathrm{uterine}$ epithelium, $\mathrm{mv}=$ maternal vessel, $\mathrm{st}=$ syncitial trophoblast, $\mathrm{ct}=$ cellular trophoblast, and $\mathrm{mbc}=$ maternal blood channel. Adapted from Leiser and Kaufmann (1994). 
In the guinea-pig, a specific carrier-mediated calcium influx on both sides of the trophoblast has been demonstrated, asymmetric between the maternal and fetal sides. A further asymmetry was observed in the backflux of transported calcium which was generally greater towards the fetal than to the maternal side.

The asymmetries in unidirectional influx into the trophoblast and rapid backflux indicated a mechanism by which the net transfer of calcium from maternal to the fetal circulation is maintained. Furthermore, the two sides of the placenta exhibited an asymmetry in both influx and efflux, which could result in a net transfer of calcium to the fetal circulation and the maintenance of the fetal-tomaternal calcium gradient. These investigations concluded that an asymmetry of placental calcium transfer was present in the guinea-pig [11,13], rat [11], and sheep $[3,6,14]$.

Transplacental calcium gradient in human varies between $1.12+/-0.06 \mathrm{mM}$ to $2.12+/-0.14 \mathrm{mM}$, in sheep 1.40 $+/-0.11 \mathrm{mM}$ to $2.59+/-0.04 \mathrm{mM}[15]$, in rat $2.42+/-$ $0.25 \mathrm{mM}$ and, in pig $0.79+/-0.03 \mathrm{mM}$ to $2.53+/-0.02$ $\mathrm{mM}[6]$.

Placental transfer of calcium in rabbit from dam to fetus was $10.3+/-3.9 \mathrm{mg}$. These results are considered low and probably due to 1 ) the effect of intraperitoneally administered sodium pentobarbital; 2) a significant return of calcium from fetus to dam during the experimental period; 3) a marked time lag in calcium movement from maternal plasma through the placenta to the fetus; and 4) a non-uniform specific activity of the maternal plasma calcium pool [16].

In experiments on the bovine placenta by [17], the authors identified the calbindin-D9k (CaBP9k) in both maternal (caruncular) and fetal (cotyledonary) epithelium. These authors suggested that CaBP9k is present in the epitheliochorial placenta of the cow (Figure 1) playing a role in the maternofetal calcium transport by functioning as an intracellular transport protein for calcium, as is hypothesized for the rodent placenta. As has been demonstrated in sheep and goats [18] the increasing demand for fetal calcium in the second half of pregnancy correlates well with a major increase of $\mathrm{CaBp} 9 \mathrm{k}$ only in the interplacentomal trophoblast. This finding indicates the important role of this region in active calcium transport in the ruminant placenta.

The equine placenta, i.e. epitheliochorial placenta (Figure 1) comprises two main structural components: flat areolae and microcotyledons [10]. The areolae surround and separate thousands of discrete microcotyledons [19]. In the equine placenta, calcium and iron are transferred by the same fetal areolar cells but by considerably different mechanisms. As in ruminants, the transport of calcium across the maternal uterine glandular epithelium to the areolar lumen seems to be associated with 9kCalcium binding protein (calbindin, 9CBP). This is supported by the evidence that in the equine placenta 9CBP is found only in the maternal glands and in the fetal areola cells $[18,19]$. Calcium is transferred through the cytoplasm with 9CBP while iron and its carrier protein (uteroferrin) is always membrane-bound during uptake via a vesicle or lysosome [19]. Several aspects about carriers or channels, the magnitude of influx/backflow and the mechanisms of calcium transport across the equine placenta remain unknown and need study to increase our knowledge on how to deal with equine diseases such as mineral deficiencies during pregnancy.

The control of the net transplancental calcium fluxes lies primarily with the fetus. It is the level and control of the CaBP9k protein in the trophoblast that are most important, and in the cow the transport of calcium from the maternal blood to the interplacental uterine lumen could be mainly passive, driven by trophoblast demand. It is hypothesized that CaBP9k-dependent active calcium transport across the placental barrier in the cow occurs by facilitated diffusion and is restricted to interplacentomal trophoblast cells, as demonstrated in other ruminants [17]. Nevertheless, contradictory information regarding the function of CaBP9k in the placental binucleate cells and uterine epithelium was reported by [20] who stated that "this protein is unique to the cow and is unlikely to be involved in calcium transport".

On the other hand, several reports have shown that in the human placenta the syncytiotrophoblast represents the specialized unit that is directly involved in fetal supply with essential nutrients, such as lipids, amino acids, and calcium. The human fetus accumulates about 25 - 30 $\mathrm{g}$ of calcium over the course of pregnancy [2,5,12,21-23].

Even though all the species studied have demonstrated a placental gradient of ionized calcium concentrations in plasma, with the fetal concentrations prevailing, the relative flux of calcium ions in either direction through the placenta varies between species. It has been suggested that these differences are directly related to the degree of skeletal maturity of the neonate. For example, in ruminants which have a well developed neonatal skeleton, bone accretion is maximized and consequently reduced placental transport of calcium produces signs of rickets [9], whereas in fetal mice [7] if depletion of PTHrP occurs, the placental calcium transport to the maternal level is not accompanied by a reduction in total body calcium. From these observations, it has been concluded that animals of small body size, like the mouse have a compensatory process of calcium diffusion as a result of the lower plasma ionized calcium concentration in the fetus. So, a reduction in calcium flux from mother to fetus, may lead a compensatory reduction in diffusion flux of calcium ions back to the mother [9]. 


\section{WHAT ARE THE MAGNITUDES AND PROFILES OF ELECTROCHEMICAL GRADIENTS ACROSS THE PLACENTA?}

Even though it has been proved in a number of studies that the transplacental gradient always advantages the fetus, the dependency of fetal blood calcium concentration upon the maternal value is not constant and varies with the species. Bawden and Wolkoff (cited by [6]) found no response of fetal blood calcium to acute maternal calcium infusion in sheep

In sheep there is evidence that prolactin may stimulate and calcitonin reduce net maternofetal calcium transfer [24]. [6] studying the fetally perfused sheep placenta, suggested that PTHrP may stimulate calcium transfer. In all species studied, placental calcium transport appears to reflect chronic, as opposed to acute, changes in maternal calcemia. In cows, the metabolism of calcium in the fetus followed the same general pattern as in the dam with an increased bone uptake resulting from additional skeletal demands for growth and possibly from an increased exchange capacity. [25] showed that immediately after $\mathrm{Ca}^{2+}$ ions are introduced into the cow's blood, they are removed by several competing process which include: 1) mixing with the body fluids and deposition in the soft tissues of the dam, 2) deposition in the bones of the dam and 3) transfer across the placenta to reach the fetal blood with subsequent deposition in the fetal soft tissues and bones. This process occurs in few minutes. However, even though calcium apparently moves across the cow placenta relatively freely, equilibration of calcium ions in the blood of fetus and dam was not observed until some time after equilibrium between blood and soft tissues of the dam had been reached, which suggests the existence of some degree of barrier.

In pig, small increases in the fetal plasma calcium concentration occurred in response to marked acute increases in maternal calcemia. On the other hand, acute decrease of calcium concentration in the maternal plasma resulted in very inconsistent variations in fetal plasma calcium concentrations. Many authors have shown that hyper and hypo-calcemia in the mother change calcium transfer through the placenta [26].

\section{WHAT PROPORTIONS OF THE FLUXES ARE EXTRACELLULAR AND WHAT PROPORTIONS TRANSCELLULAR, USING TRANSPORT PROTEINS (CARRIERS AND CHANNELS) IN THE TROPHOBLAST PLASMA MEMBRANE?}

The uptake of calcium by the chorionic trophoblast of the ovine placenta is time-dependent, with no significant increases in the rate of uptake throughout most of the last third of pregnancy. Uptake process is also temperature-dependent which indicates that it is not simply passive but is again dependent on maternal calcium concentration. The involvement of a specific calcium channel was indicated by the studies of Jones et al. [27] who demonstrated significant inhibition of uptake by all of traditional L-type calcium channel blockers.

Information regarding cellular mechanisms and molecular events associated with calcium transport in the placenta is scarce. It is only known that the presence of free calcium in the trophoblast is like that in any other cell. From there, the placental trophoblast has the particular task of delivering calcium to the fetus in addition to maintaining a low intracellular calcium concentration.

If the intracellular concentration of free calcium is lower than in the blood, then it can be assumed that the plasma membrane possesses an energy-dependant extrusion mechanism. Additionally, since calcium movement is mostly directed toward the fetus, this active extrusion should be localized on the membrane facing the fetus. These membrane mechanisms may interact with placental cytoplasmic factors in the homeostasis of fetal calcium in the guinea-pig. The use of inhibitors and hormones such as vitamin D and its metabolites should permit further characterization of calcium transport.

In the rat also, since the ionic equilibrium across the placenta seems not to be influenced by electrical forces, the concentration difference of free calcium across the placenta suggests that the maternofetal transport of calcium is active. By analogy to other calcium-transporting epithelial barriers it can be assumed that placental transport of calcium is transcellular, with passive entry of calcium into the trophoblast from one side being followed by active extrusion from the other side [11].

Guidotti et al. (1978) cited by [11] has established that, in the rat, the concentration of calcium in fetal plasma depends on the balance between the active transport by the placenta and uptake of calcium by fetal tissues. The authors considered it improbable that fetal homeostasis of calcium could be maintained without the calcium transport across the placenta being regulated. According to [11] bidirectional diffusion across the placenta tends to maintain equilibrium of the calcium concentrations between fetal and maternal plasma and may be considered an important aspect of fetal homeostasis. The authors state that "When it is high relative to the net flux, the concentration of the substance in fetal plasma will closely follow that on the maternal side of the placenta, and fetal homeostasis of the substance may be maintained by maternal homeostatic mechanisms". However the same authors concluded that the diffusional movement of calcium across the rat placenta represented only a small fraction of the net flux of calcium. As a consequence, the trans- 
placental diffusion of calcium can hardly contribute significantly to maintaining fetal calcium homeostasis in this species.

\section{WHAT IS THE MOST COMMON ROUTE OF CALCIUM TRANSPORT IN THE DIFFERENT SPECIES?}

Calcium is transported across the placenta, whether it is epitheliochorial (horse and pig, Figure 1(a)), synepitheliochorial (ruminants, Figure 1(b)), endotheliochorial (carnivores, Figure 1(c)) or hemochorial (rodents, bats, primates, and human, Figures 1(d)-(f)) $[6,10]$.

All the nutrients and minerals required by developing ovine fetus must pass from the maternal circulation through either maternal glandular or luminal epithelium (interplacentomal) or fetomaternal syncytiotrophoblast (placentomal) and finally across the trophoblast to enter in the fetal circulation. The much higher expression of CaBP9k in glandular and interplacentomal trophoblast epithelia than in the placentomal epithelia suggests that calcium transport in ruminants may be concentrated in the interplacentomal areas [18].

The calcium probably diffuses passively or through specific channels along its electrochemical gradient. It must enter the fetal blood by means of an energy-consuming process, which might require the intervention of a calcium pump, calcium binding proteins and possibly intracellular messengers such as cyclic AMP, all of which are present in the syncytial cell.

[26] demonstrated in sheep that fetal parathyroid glands secrete a substance capable of stimulating the placental transfer of calcium to the fetus, and that the secretion of that substance was depressed by fetal hypercalcemia. According to [6] establishing the correlation between maternal and fetal plasma calcium concentrations is usually low and even absent in some species. The author concluded that hormones such as $1.25-(\mathrm{OH}) 2$ vitamin D3 are responsible for controlling calcium delivery to the fetus, and that this control exists on the fetal side. Furthermore, the author concluded that the absence of maternal vitamin $\mathrm{D}$ has only general effects on fetal growth that is partly dependent on maternal calcium intake, meaning that the absence of maternal vitamin D could not specifically affect fetal mineral homeostasis. So to speak, vitamin D-dependent calcium binding protein probably plays an important role in calcium transport through placenta since it is reported that specific antibodies decrease calcium uptake by microsomal vesicles.

\section{IS THERE ANY REPORTED MECHANISM OF IONIZED $\mathrm{Ca}^{2+}$ TRANSFER ACROSS THE PLACENTA?}

Several mechanisms are mentioned by which mineral transfer occurs: solvent drag (in perfused human placental cotyledon); simple diffusion, along concentration and electrical gradients; and transcellular transfer which utilizes transport of proteins through microvillus or basal membranes of the syncytiotrophoblasts [28].

Although calcium transport against a gradient through the placenta is a good example of active electrolyte transport, the mechanisms involved are poorly understood $[6,23,29]$.

However, in chicks, four general steps have been suggested: 1) calcium influx across the apical plasma membrane, cytosolic transit (which may involve a calciumbinding protein to keep calcium low and help translocation of calcium) and, finally, removal of calcium through a basolateral pumping mechanism; 2) an extracellular passive diffusion mechanism where calcium accumulates at the cell surface and transport occurs along cellular interstices [30]; 3) the use of sub-cellular organelles, particularly mitochondria, to sequester large amounts of cellular calcium to be released into the cytosol, and then pumped across the basolateral membranes [30]; 4) modified endocytosis-like process involving calcium accumulation at the apical surface of the cells followed by internalization and vesicle-mediated delivery to the basolateral surface [6].

Thus, calcium transport by the chick chorioallantoic membrane (CAM) is a regulated process that supplies the developing embryo with eggshell calcium to be used in the bone development. Transepithelial transport by the CAM has been shown to be active and unidirectional. It is clear that although CAM ectoderm cells quickly internalize large amounts of calcium, it has to be sequestered away from the cytosol [30].

Calcium transport across both maternal and fetal placental epithelium in the sheep is reportedly divided into three major steps: 1) passage through the basolateral membrane of the gland cell and the apical membrane of the trophoblast (mediated by one or more calcium channels which may include ionized $\mathrm{Ca}^{2+}$-release-activated channel (ICRMC), second-messenger-operated channels and even the G-protein-operated channel; 2) binding mechanisms that permit the maintenance of a low cytosolic concentration of free calcium whilst the bound calcium is transported across the cell and 3) extrusion mechanisms in the apical plasma of the gland cells, and at the basolateral plasma of the trophoblast, to deliver calcium to the fetal circulation [27].

$[15,31]$ only state that calcium is transported actively (through the plasma membrane) against a concentration gradient, from the maternal circulation to the fetal circulation across the placenta. This statement was fully supported by [8].

According to $[1,12]$ calcium transport across the human placenta could plausibly involve three main steps: 1 ) 
diffusion into the trophoblast from maternal plasma down an electrochemical gradient through epithelial calcium channels of the transient receptor potential (TRP) gene family; 2) transfer across the trophoblast cytoplasm bound to the calcium binding protein calbindin-D9K [17] and 3) active extrusion into the fetal compartment via plasma membrane $\mathrm{Ca}^{2+}$ ATP-ase (PMCA) localized to the fetal facing basal plasma membrane [7].

[2] revealed in the human term placenta, the presence of high-affinity $\mathrm{Ca}^{2+}$-stimulated ATPase activity in microvillous membrane (MVM-maternal facing) of which was capable of transporting $\mathrm{Ca}^{2+}$ against its gradient in MVM vesicles and to have biochemical characteristics resembling those of Plasma membrane Ca-ATPase (PMCA) which also has high affinity for free $\mathrm{Ca}^{2+}$. In the same investigation a new model for the transport of calcium across the human syncytiotrophoblast was proposed considering the presence of an important fraction of PMCA in MVM.

\section{IS THERE ANY CONTROL OF PLACENTAL IONIZE Ca ${ }^{2+}$ TRANSFER AND IF SO, HOW IT IS BROUGHT ABOUT?}

The regulation of calcium in the placenta is made by calcium channels/transporters, components that are distributed in specific places and reveal physiological properties (intrusion and extrusion of calcium) in the placenta. The maintenance of low intracellular concentrations of calcium is crucial for cell survival as well as for its function as second messenger [1]. These data suggested that calcium is transferred to the fetus by means of active processes which may be regulated by the placenta itself.

Transplacental transfer of calcium in the guinea-pig cannot be used to characterize specific calcium-transport mechanisms, even through the trophoblast measurements were provided by the single-circulation or paired-tracer technique [13]. Several evidences suggested that in late gestation of mammals, calcium is transported across the placenta from mother to fetus against a concentration gradient [15]. So, it has been showed by $[15,16]$ that this transplacental calcium gradient in favor of the fetus is not a result of increased calcium-binding capacity of fetal serum proteins nor dependant of the presence of the fetus.

During pregnancy, the syncytiotrophoblast of the human placenta (Figure 1) transfers approximately $30 \mathrm{~g}$ of calcium from the mother to fetus, especially in late gestation where calcium transport through different channels must increase in response to the demands of accelerating bone mineralization of the fetus [8]. In the rat, the transcellular transport across the trophoblast is likely to take place against a background of paracellular diffusion. Since there is a lack of tight correlation between the plasma calcium concentration in the fetus and mother, it is assumed that some control mechanism of calcium transport exists.

The control of placental transfer of calcium is probably on the fetal side of the placenta. This observation was concluded after umbilical perfusion experiments have done in the guinea-pig fetuses, where calcium transport against a gradient (from maternal to fetal circulation) is observed only when the perfusate consists of plasma or whole blood. On the other hand, when the perfusate simply contained electrolytes with glucose and fluorocarbon as oxygen carrier, no calcium transport was observed [6]. Even though the placental barrier in the rat and in the guinea-pig differ histologically because of the number of trophoblast layers (three in the rat placenta and only a single layer in the guinea pig placenta), the permeability properties of these placentae are very similar, which probably means that the number of trophoblast layers in the placenta has little influence on the transplacental diffusion of hydrophilic molecules [11].

In sheep, fetal death was followed by an increase in the rate of transfer of calcium from the fetus to the mother along the concentration gradient [14]. These observations suggested that fetal blood plays a vital role in the transport of calcium according to fetal requirements. This hypothesis is reinforced by [6] findings where it is assured that active transport occurs in the membrane facing the fetus. Even though it is proven that calcium is a major requirement for developing embryos, the distribution of $\mathrm{Ca}^{2+}$ ATPase differs in the uterus of oviparous and viviparous species. The changes are correlated with increasing placental complexity in viviparous [32].

Several studies demonstrated that the presence of $\mathrm{Ca}^{2+}$ ATPase pumps in the shell glands is responsible for production of the calcareous and non-calcareous part of the eggshell in oviparous species [32]. These results were strongly supported by $[33,34]$ studies in Japanese quail and domestic chicken respectively.

Calcium transport from the eggshell to the developing chick embryo is carried out by the ectoderm cells of the chick CAM. It has been proven that during the development of most mammals, placenta serves as tissue providing calcium. In oviparous species the calcium supply function of the placenta is accomplished by the embryonic [30].

Afterwards, [32] showed that the transport of calcium (that will be stored in the egg-yolk to be used by the embryo) across the placenta was made of $\mathrm{Ca}^{2+}$ ATPase pumps in the earlier phase of pregnancy. For this reason, these authors explained that the evolution of placenta accordingly to the evolution of the species, from the simple placenta to the most complex structure which probably are better able to supply nutrients to the embryo, results in $\mathrm{Ca}^{2+}$ ATPase pumps retention into the uterine 
epithelium during the later stages of pregnancy when there is a greatest demand for calcium by the embryo.

\section{CONCLUSION}

The literature indicates that the concentration of calcium in the fetal blood serum is significantly higher than in the maternal serum. The syncytiotrophoblast layer of the placenta is the major site of exchange of nutrients and gases between mother and fetus. The transport of calcium is essential for normal fetal growth and development. An active transport mechanism for calcium transfer exists and the amount transferred varies with species. Calcium transport across the placenta is bidirectional with species variations in magnitudes of the maternofetal and fetomaternal fluxes and the consequent net maternofetal flux. Adequate levels of calcium are essential throughout pregnancy but particularly in the last third of gestation due to its role in the growth and development of the fetus. In many circumstances this is only achieved by calcium supplementation. Many aspects of the exact control and mechanism of calcium transfer remain to be resolved. In order to clarify these aspects different animal models have been used to better understand the effects of the calcium, especially when it is disturbed in both fetus and mother during the gestation. Among the different species particular aspects of the calcium transport across the placenta have been demonstrate and these results can help to solve health problems especially in regard to the fetus development during the pregnancy.

\section{REFERENCES}

[1] Belkacemi, L., Bédard, I., Simoneau, L. and Lafond, J. (2005) Calcium channels, trasporters and exchangers in placenta: A review. Cell Calcium, 37, 1-8. doi:10.1016/j.ceca.2004.06.010

[2] Marin, R., Riquelme, G., Godoy, V., Diaz, P., Abad, C., Caires, R., Proverbio, T., Pinero, S. and Proverbio, F. (2008) Functional and structural demonstration of the presence of Ca-ATPase (PMCA) in both microvillous and basal plasma membranes from syncytiotrophoblast of human term placenta. Placenta, 29, 671-679.

doi:10.1016/i.placenta.2008.06.003

[3] Ramberg Jr., C.F., Delivoria-Papadopoulos, M., Crandall, E.D. and Kronfeld, D.S. (1973) Kinetic analysis of calcium transport across the placenta. Journal of Applied Physiology, 35, 682-688.

[4] Lester, G.E. (1986) Cholecalciferol and placental calcium transport. Federation Proceedings, 45, 2524-2527.

[5] Smith, C.H. and Moe, A.J. (1992) Nutrient transport pathways across the epithelium of the placenta. Annual Reviews of Nutrition, 12, 183-206. doi:10.1146/annurev.nu.12.070192.001151

[6] Brunette, M.G. (1988) Calcium transport through the placenta. Canadian Journal of Physiology and Pharmacol- ogy, 66, 1261-1269. doi:10.1139/y88-207

[7] Hosking, D.J. (1996) Calcium homeostasis in pregnancy. Clinical Endocrinology, 45, 1-6.

[8] Husain, S.M. and Mughal, M.Z. (1992) Mineral transport across the placenta. Archives of Disease in Childhood, 67, 874-878. doi:10.1136/adc.67.7_Spec_No.874

[9] Care, A.D. (1997) Fetal calcium homeostasis. Equine Veterinary Journal, 24, 59-61.

[10] Leiser, R. and Kaufmann, P. (1994) Placental structure: In a comparative aspect. Experimental Clinical Endocrinology, 102, 122-134. doi:10.1055/s-0029-1211275

[11] Stulc, J. and Stulcová, B. (1986) Transport of calcium by the placenta of the rat. Journal of Physiology, 371, 1-16.

[12] Bond, H., Dilworth, M.R., Baker, B., Cowley, E., Jimenez, A.R., Boyd, R.D.H., Husain, S.M., Ward, B.S., Sibley, C.P. and Glazier, J.D. (2008) Increased maternofetal calcium flux in parathyroid hormone-related protein-null mice. Journal of Physiology, 586, 2015-2025. doi:10.1113/jphysiol.2007.149104

[13] Sweiry, J.H. and Yudilevich, D.L. (1984) Asymmetric calcium influx and efflux at maternal and fetal sides of the guinea-pig placenta: Kinetics and specificity. Journal of Physiology, 355, 295-311.

[14] Weatherley, A.J., Ross, R., Pickard, D.W. and Care, A.D. (1983) The transfer of calcium during perfusion of the placenta in intact and thyroparathyroidectomized sheep. Placenta, 4, 271-278. doi:10.1016/S0143-4004(83)80006-X

[15] Delivoria-Papadopoulos, M., Battaglia, F.C., Bruns, P.D. and Meschia, G. (1967) Total, protein-bound and ultrafiltered calcium in maternal and fetal plasmas. American Journal of Physiology, 213, 363-366.

[16] Twardock, A.R. and Austin, M.K. (1970) Calcium transfer in perfused guinea pig placenta. American Journal of Physiology, 219, 540-545.

[17] Reiswig, J.D., Frazer, G.S. and Inpanbutr, N. (1995) Calbindin-D9k expression in the pregnant cow uterus and placenta. Histochemistry Cellular Biology, 104, 169-174. doi:10.1007/BF01451576

[18] Wooding, F.B.P., Morgan, G., Jones, G.V. and Care, A.D. (1996) Calcium transport and the localization of calbidinD9K in the ruminant placenta during the second half of pregnancy. Cell Tissue Research, 285, 477-489. doi:10.1007/s004410050664

[19] Wooding, F.B.P., Morgan, G., Fowden, A.L. and Allen, W.R. (2000) Separate sites and mechanisms for placental transport of calcium, iron and glucose in the equine placenta. Placenta, 21, 635-645. doi:10.1053/plac.2000.0550

[20] Nikitenko, L., Morgan, G., Kolesnikov, S.I. and Wooding, F.B.P. (1998) Immunocytochemical and in situ hybridization studies of the distribution of Calbidin D9k in the bovine placenta throughout pregnancy. The Journal of Histochemistry \& Cytochemistry, 46, 679-688. doi: $10.1177 / 002215549804600513$

[21] Kamath, S.G., Haider, N. and Smith, C.H. (1994) ATP independent calcium transport and binding by basal plasma membrane of human placenta. Placenta, 15, 147-155. doi:10.1016/S0143-4004(05)80451-5 
[22] Prentice, A. (2000) Maternal calcium metabolism and one mineral status 1-3. The American Journal of Clinical $\mathrm{Nu}$ trition, 71, 1312S-1316S.

[23] Moreau, R., Daoud, G., Bernatchez, R., Simoneau, L., Masse, A. and Lafond, J. (2002) Calcium uptake and calcium transporter expression by trophoblast cells from human term placenta. Biochimica et Biophysica Acta, 1564, 325-332. doi:10.1016/S0005-2736(02)00466-2

[24] Robinson, N.R., Sibley, C.P., Mughal, M.Z. and Boyd, R.D.H. (1989) Fetal control of calcium transport across the rat placenta. Pediatric Research, 26, 109-115. doi:10.1203/00006450-198908000-00008

[25] Plumlee, M.P., Hansard, S.L., Comar, C.L. and Beeson, M.W. (1952) Placental transfer and deposition of labeled calcium in the developing bovine fetus. American Journal of Physiology, 171, 678-686

[26] Care, A.D. (1986) Placental transfer of calcium to the ovine fetus and its regulation. Proceedings of the Nutrition Society, 46, 321-329. doi:10.1079/PNS19870040

[27] Jones, G.V., Taylo, K.R., Morgan, G., Wooding, F.B.P. and Care, A.D. (1997) Aspects of calcium transport by the ovine placenta: Studies based on the interplacentomal region of the chorion. Placenta, 18, 357-364. doi:10.1016/S0143-4004(97)80070-7

[28] Kane, S.V. and Acquah, L.A. (2009) Placental transport of immunoglobulins: A clinical review for gastroenterologists who prescribe therapeutic monoclonal antibodies to women during conception and pregnancy. American Journal of Gastroenterology, 104, 228-233. doi:10.1038/ajg.2008.71

[29] Abrams, S.A. (2007) In utero physiology: Role in nutrient delivery and fetal development for calcium, phosphorus, and vitamin D1-4. The American Journal of Clinical Nutrition, 85, 604S-607S.

[30] Akins, R.E. and Tuan, R.S. (1993) Transepithelial calcium transport in the chick chorioallantoic membrane. II. Compartmentalization of calcium during uptake. Journal of Cell Science, 105, 381-388.

[31] Pitkin, R.M. (1985) Calcium metabolism in pregnancy and the perinatal period. American Journal of Obstetrics and Gynecology, 151, 99-109.

[32] Herbert, J.F., Lindsay, L.A., Murphy, C.R. and Thompson, M.B. (2006) Calcium transport across the uterine epithelium of pregnant lizards. Herpetological Monographs, 20, 205-211. doi:10.1655/0733-1347(2007)20[205:CTATUE]2.0.CO;2

[33] Yamamoto, T., Yamamoto, H. and Ozawa, H.N. (1985) Histochemical studies of Ca-ATPase, succinate and NAD ${ }^{+}$dependent isocitrate dehydrogenases in the shell gland of laying Japanese quails: With special reference to calciumtransporting cells. Histochemistry, 83, 221-226. doi:10.1007/BF00953987

[34] Wasserman, R.H., Smith, C.A., Smith, C.M., Brindak, M.E., Fullmer, L.C.S., Krook, J.T. and Penniston, R.K. (1991) Immunohistochemical localization of a calcium pump and calbindin-D28k in the oviduct of the laying hen. Histochemistry, 96, 413-418. doi:10.1007/BF00315999 